\title{
Interleukin-17 cytokine signalling in patients with asthma
}

\author{
Anders Lindén ${ }^{1,2}$ and Barbro Dahlén ${ }^{2}$
}

\author{
Affiliations: \\ 'Unit for Lung and Airway Research, Institute of Environmental Medicine, Karolinska Institutet, Stockholm, \\ Sweden. \\ ${ }^{2}$ Lung Allergy Clinic, Dept of Medicine at Karolinska University Hospital, Stockholm, Sweden.

\section{Correspondence:} \\ Anders Lindén, Institute of Environmental Medicine, Karolinska Institutet, PO Box 210, SE-171 77 Stockholm, \\ Sweden. \\ E-mail: anders.lindenaki.se
}

ABSTRACT Asthma remains a global health problem and, therefore, more effective pharmacotherapy is needed. This is particularly true for chronic and severe asthma. In these clinical phenotypes, chronic inflammation involving neutrophils is likely to play a pathogenic role, making it interesting to target cytokine signalling involved in the accumulation of neutrophils. Therefore, it is of interest that the archetype T-helper 17 cell cytokine interleukin (IL)-17A, perhaps also IL-17F, controls neutrophil accumulation, mucus secretion, macrophage mobilisation and smooth muscle reactivity in various experimental airway models. However, much less is known about the involvement of signalling via IL-17 cytokines in humans with asthma. Existing evidence suggests that these cytokines are released from several types of immune cells in asthma and, for IL-17A, there is a local increase associated with disease severity, with the mobilisation of neutrophils and smooth muscle cells locally in the airways. Even though the causative role of IL-17 cytokines remains unclear, there is potential for clinical utility in targeting IL-17A specifically in patients with moderate-to-severe asthma and high reversibility. There is a need for new and well-powered clinical investigations of signalling via IL-17 cytokines in this clinical phenotype.

○ @ERSpublications

There is potential utility for drugs targeting IL-17 cytokine signalling in a sub-group of patients with asthma http://ow.ly/wPw5p

Support statement: The author received funding from the Karolinska Institutet (Stockholm, Sweden), the Swedish HeartLung Foundation (Stockholm) and King Gustaf V and Queen Victoria's Freemason Foundation (Stockholm), which made this work possible. No funding was obtained from the tobacco industry and the authors have no competing financial interest.

Conflict of interest: None declared. 


\section{Introduction}

Asthma in humans constitutes a global health problem, affecting more than 300 million people of all ages [1]. Despite substantial progress in terms of optimising existing pharmacotherapeutic principles, therapy remains insufficient and few really novel principles have been developed during recent years. This is particularly true for severe asthma, which accounts for $\sim 10 \%$ of all patients with this more or less reversible obstructive airway disease [1]. Thus, there is an unmet global need for improved pharmacotherapy and this means that there is a matching need for more research into pathogenic mechanisms and novel molecular targets.

The fact that not only eosinophils, but also neutrophils (synonymous to neutrophilic granulocytes or polymorphonuclear granulocytes), are mobilised in certain clinical phenotypes of asthma has been recognised [2-6]. This is also true for the fact that clinically important phenotypes among adult patients lack signs of true allergy. It is now also clear that the T helper (Th) 2 cell paradigm is insufficient to explain the whole variety of clinical phenotypes for asthma [2-6]. However, even though these more recent insights have taken the mechanistic understanding of asthma to a new level, they have not yet provided us with new pharmacotherapeutic tools.

Given the lack of sufficient therapy against asthma and due to recent and ongoing trials on drugs targeting the archetype Th17 cytokine interleukin (IL)-17A (synonymous to IL-17), this review will focus on summarising and scrutinising the accumulating body of evidence from (human) patients in whom IL-17A, and additional members of the IL-17 cytokine family (IL-17 cytokines), are actively involved in the pathogenesis of asthma. The preceding work in various animal and cell models, including the experimental evidence from models of allergic asthma, has been extensively reviewed and presented previously [7-14].

\section{Original hypothesis on neutrophil accumulation}

The original hypothesis that the heterodimeric cytokine IL-17A contributes to excess accumulation of neutrophils in the airways of patients with asthma was communicated more than a decade ago [7-9]. This hypothesis was initially based merely on plain experimental studies that documented the neutrophil mobilising potential of IL-17A protein in animal airway models in vivo plus models of human bronchial epithelial and venous endothelial cells in vitro [15-17]. In essence, the results of these early experimental studies indicated that IL-17A recruits and accumulates neutrophils indirectly into the airways by inducing the transcription and release of neutrophil mobilising cytokines from structural airway cells (fig. 1). These early studies and the subsequent studies on "pro-inflammatory effects" of IL-17A demonstrated that the IL-17-induced accumulation of neutrophils is also associated with an increase in proteolytic enzymes, including neutrophil elastase and matrix metalloproteinase- $9[17,18]$. The initial evidence for the actual involvement of IL-17A in patients with asthma was published several years later by MOLET et al. [19] and the observations in patients thereafter rapidly obtained support in two subsequent studies [20, 21].

\section{Variety of functional effects \\ Neutrophils and macrophages}

During the past 5 years, several experimental studies have indicated that IL-17A can do more than merely stimulate the accumulation of neutrophils and the release of their proteases in the airways (fig. 1). Thus, there is now experimental evidence from cell and animal models suggesting that IL-17A may stimulate the recruitment of macrophage precursor cells (i.e. monocytes), macrophage survival and phagocytosis of particles [22, 23]. Moreover, IL-17A can stimulate neutrophil apoptosis and macrophage phagocytosis of aged neutrophils [23]. In other words, IL-17A may exert "anti-inflammatory effects" that are important for ascertaining the efferocytosis of neutrophils and, thus, the resolution of the innate immune response (fig. 2).

It was recently demonstrated in cell and animal models that IL-17A is important for providing negative feedback on the release of the Th17 regulator IL-23 from macrophages (fig. 2). Thus, it seems feasible that there is a mammalian mechanism to avoid excess signalling through the IL-23/IL-17A axis. Hypothetically, this type of mechanism can serve to maintain a balanced control of neutrophil turn-over for the purpose of preserving an effective antibacterial host defence response without tissue damaging activity [24]. The proposed feedback mechanism is in line with the observation in an animal model that IL-17A also exerts negative feedback on the expansion of IL-17-producing T-cells [25].

\section{Other cells involved in asthma}

In addition to its well-documented effects on macrophages and neutrophils, there is evidence from animal models and isolated human bronchi in vitro that IL-17A stimulates airway responsiveness [26, 27]. In line with an indirect role for IL-17A in bronchoconstriction, CHANG et al. [28] originally showed that IL-17A promotes the proliferation and survival of human airway smooth muscle cells in vitro, with a similar relative impact on cells from patients with asthma and control subjects. In further support of an indirect role for 
IL-17A in bronchoconstriction and/or hyperreactivity, CHANG et al. [29] have also shown that IL-17A increases the migration of human airway smooth muscle cells in vitro. Compatible with a more specific role in allergy, SCANLON et al. [30] demonstrated that IL-17A stimulates the production of the B-cell chemokine CCL28 in human airway epithelial cells. These authors also demonstrated that the induced CCL28 release increases the chemotaxis of IgE-containing B-cells in vitro. In addition, IL-17A stimulates the gene expression for the mucin MUC5B in primary bronchial cells harvested from healthy donors and cultured in vitro, which is compatible with a role for IL-17A in airway hypersecretion, a hallmark of acute, severe asthma [31, 32]. Moreover, bronchial epithelial cells from patients with asthma and airway hyperresponsiveness cultured in vitro respond to IL-17A with secreted phospholipase A2 group X, indicating a feasible link between IL-17A and the production of lipid mediators in asthma [33]. However, even though the reported effects of IL-17A are intriguing from a pathogenic point of view, there is a general need to evaluate how relevant these effects are in causative terms for asthma patients with or without allergy.

\section{Local involvement}

A substantial body of clinical evidence now argues for the presence of IL-17A in the airways of patients with asthma, including data on this heterodimeric protein in bronchoalveolar lavage (BAL), sputum samples, exhaled breath condensate and bronchial tissue.

\section{Bronchoalveolar lavage samples}

In their seminal study, MOLET et al. [19] reported that the concentrations of soluble IL-17A protein in BAL were moderately increased in patients with mild asthma compared with healthy control subjects. In their study, a substantial fraction of the asthma patients displayed allergen sensitisation and some were exsmokers but no patient had a history of heavy smoking, was treated with glucocorticoids or had a recent respiratory infection. Thus, it seems unlikely that the presence of IL-17A would be due to a mere activation

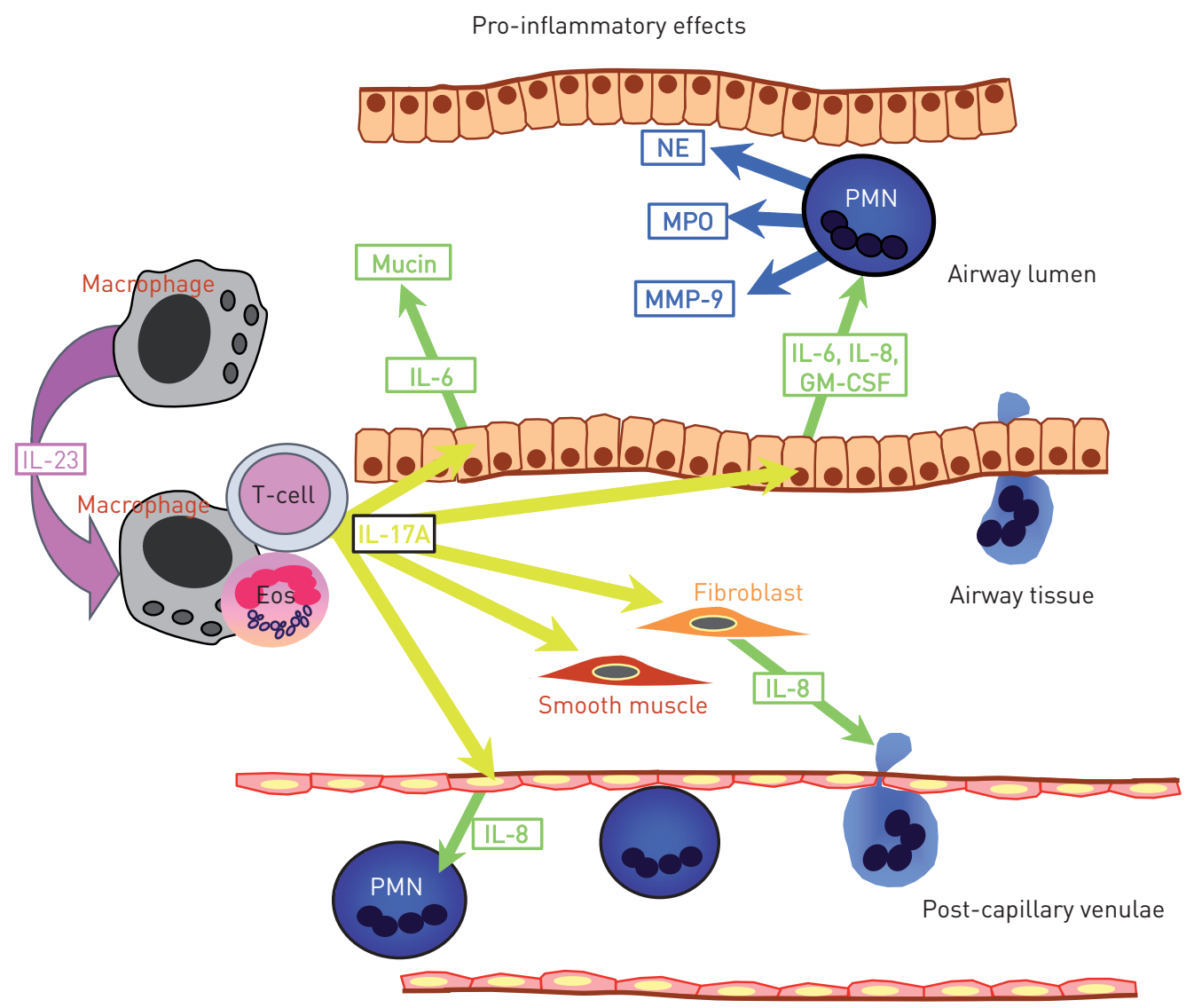

FIGURE 1 Overview of the pro-inflammatory effects caused by interleukin (IL)-17A in experimental studies that are of hypothetical relevance for the pathogenesis of asthma. These include the accumulation of neutrophils and macrophages. Inflammatory cells such as T-cells, macrophages, polymorphonuclear neutrophils (PMN) and eosinophils (Eos) are indicated, as are effector molecules such as matrix metalloproteinase (MMP)-9, myeloperoxidase (MPO), neutrophil elastase (NE) and mucins. GM-CSF: granulocyte-macrophage colony-stimulating factor. 
of pulmonary host defence in this important study. MOLET et al. [19] also demonstrated a substantially more frequent immunoreactivity signal for IL-17A protein in BAL cells from patients with mild asthma, which was associated with a clear trend towards an increased fraction of neutrophils compared with control subjects.

\section{Sputum samples}

In support of their original findings in BAL fluid and cells, MolET et al. [19] demonstrated that the immunoreactivity for IL-17A protein is considerably more abundant in sputum cells from patients with mild asthma when compared with healthy control subjects. Similar to their findings in BAL fluid, MOLET et al. [19] also detected a trend towards an increase in sputum neutrophils that was associated with the increase in IL-17A.

In a follow-up study in a limited number of patients with mild-to-moderate asthma but without any documentation of atopy, BARCZYK et al. [34] demonstrated a trend towards an increase in luminal IL-17A protein when analysing induced sputum from patients with asthma compared with healthy control subjects. While conducting a pooled analysis of the patients with asthma plus a similarly limited number of patients with chronic bronchitis, BARCZYK et al. [34] detected a correlation between an increased concentration of IL-17A protein in induced sputum and the bronchial responsiveness to methacholine (measured as provocation concentration causing a $20 \%$ fall in forced expiratory volume in $1 \mathrm{~s}$ ). However, when comparing the hyperresponsive subgroup of patients with a healthy control group without hyperresponsiveness, the average difference in sputum IL-17A emerged as modest, even though it was statistically significant. It cannot be ruled out that the inconsistent treatment with inhaled glucocorticoids among the patients with asthma contributed to this somewhat vague outcome, given what is known about the sensitivity of the production of IL-17A to glucocorticoids from animal and cell models [35]. Along these lines, in the study by BARZYCK et al. [34] the patients with asthma did not have an increase in sputum neutrophils.

Sun et al. [36] demonstrated a substantial increase in IL-17A protein in induced sputum from patients with severe asthma compared with healthy control subjects. Notably, this increase in IL-17A was also accompanied by a substantial and corresponding increase in the fraction of neutrophils among sputum cells. Sun et al. [36] also examined patients with mild-to-moderate asthma and, when analysing IL-17A

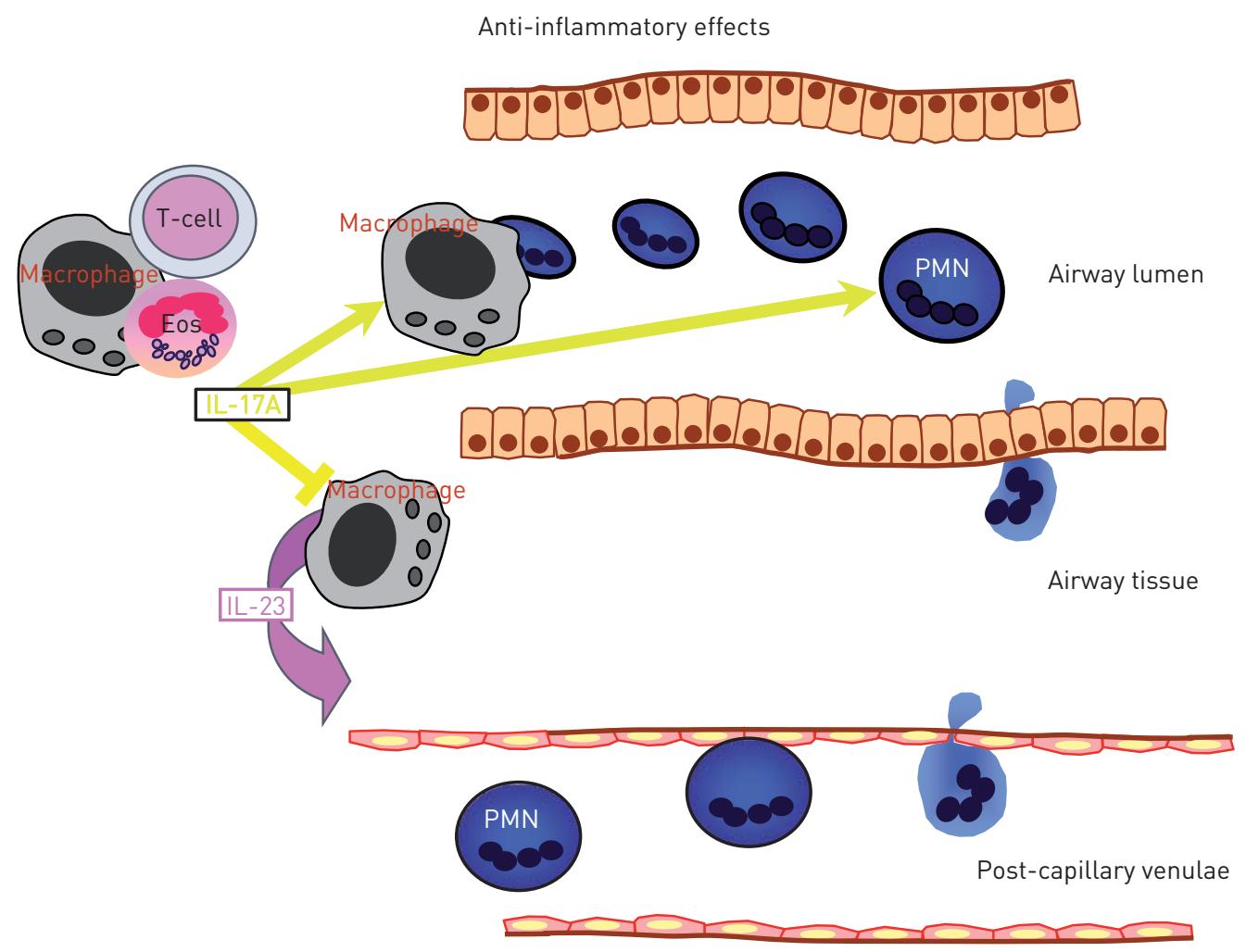

FIGURE 2 Overview of anti-inflammatory effects caused by interleukin (IL)-17A in experimental studies that are of hypothetical relevance for the pathogenesis of asthma. These include the induced apoptosis and phagocytosis of neutrophils. Inflammatory cells such as T-cells, macrophages and polymorphonuclear neutrophils (PMN) are indicated. Eos: eosinophils. 
protein in all stages of disease, they found that the increase in sputum IL-17A clearly paralleled the severity of disease. The same was true for the protein concentrations of both the neutrophil-recruiting chemokine IL-8 and the activity marker myeloperoxidase, thus illustrating the association of IL-17A with neutrophil mobilisation in asthma. Importantly, none of the patients with asthma were treated with glucocorticoids at the time of the referred investigations, were recently infected or were a smoker [36]. Moreover, when patients with all three degrees of disease severity were subsequently treated with a moderate daily dose of an inhaled glucocorticoid, this treatment reduced the concentration of IL-17A protein. This suggests that the net release of IL-17A protein in the airways of patients with asthma is actually sensitive to glucocorticoids under these conditions, contrasting with previously published data indicating a very low sensitivity to glucocorticoids in animal models $[35,37]$. However, and this may be important, the utilised glucocorticoid treatment did not markedly affect the relative number of neutrophils in induced sputum from the patients in this study.

A true correlation between IL-17A and neutrophils was demonstrated by BuLLENs et al. [38] when assessing mRNA for IL-17A in sputum cells from patients with mild and moderate-to-severe asthma. However, the increase in this mRNA was modest compared with healthy control subjects but the results did not suggest that the transcription of IL-17A is sensitive to treatment with inhaled glucocorticoids. In addition, there was a trend only towards a moderately higher level of mRNA for IL-17A in verified allergic compared with non-allergic asthma.

An unexpected link between IL-17A and a particular clinical phenotype of asthma was demonstrated by KAMINSKA et al. [39]. The authors assessed airway remodelling outcomes versus cytokine content in induced sputum and compared patients with chronic versus reversible phenotypes of severe asthma [39]. They found a higher content of luminal IL-17A protein when analysing sputum samples from patients with the reversible rather than with the chronic phenotype, but there was actually a luminal neutrophil accumulation of a similar magnitude in both phenotypes. Thus, it can be speculated that in addition to IL-17A, other mechanisms are involved in determining the luminal accumulation of neutrophils in patients with asthma, even though additional factors may have set the outcome of this particular study. First, there was inconsistent use of inhaled and oral glucocorticoids for the two study groups. Secondly, it is known that sputum sampling tends to yield higher neutrophil content in all samples and reflect more proximal conditions than BAL samples. Hypothetically, these factors may have shaded a more clear-cut difference in neutrophil accumulation in the lower airways for the patients with the reversible compared to the chronic phenotype.

DoE et al. [40] compared the concentration of IL-17A protein in the sputum of patients with asthma with that observed in patients with chronic obstructive pulmonary disease (COPD). These investigators detected higher concentrations of IL-17A protein in patients with COPD and no ongoing exacerbation compared with patients with asthma, even though the highest individual concentrations of IL-17A were detected in patients with asthma. Thus, this study indicated that there is a higher luminal concentration of IL-17A in COPD than asthma. However, the interpretation is complicated by the fact that current smokers were included among the patients with asthma in such a number that the average tobacco load exceeded that of the healthy control subjects. Moreover, former and never-smokers, but no current smokers, were included among the patients with COPD and the average tobacco load in the COPD group exceeded that in the group of smokers without COPD [40]. Finally, there was no information on the types of inhalation therapy being used by either of the patient groups and, thus, the confounding influence of glucocorticoids cannot be excluded. The key results from the study by DoE et al. [40] suggesting that there is more IL-17A in COPD than in asthma can, therefore, be questioned. These results are in need of confirmation by new studies with improved control of the confounding influence of tobacco load among the study groups.

Finally, given the question about IL-17 signalling and atopy, it is interesting to note the results from a recent study by MANISE et al. [41]. These investigators showed that the concentration of IL-17A protein in induced sputum is higher in patients with asthma plus high serum levels of IgE compared with low levels of this type [41]. As is expected, the former group also displayed a higher frequency of atopy, so a link to atopy cannot be excluded. However, the relevance of these findings in sputum remains to be verified in terms of their relevance for conditions in the peripheral airways.

\section{Exhaled breath condensate}

Matsunaga et al. [42] published a study on the concentrations of IL-17A protein in exhaled breath condensate from patients with asthma and healthy control subjects. The subjects were nonsmokers and who were not receiving any glucocorticoid treatment. In accordance with most of the studies on BAL and sputum samples, the results of this study suggest that IL-17A protein is moderately increased in patients with mild asthma, compared with healthy control subjects. It is of particular mechanistic interest that MATSUNAGA et al. [42] obtained indications that the increase in IL-17A among these patients with asthma is 
associated with a corresponding increase in IL-8, tumour necrosis factor (TNF)- $\alpha$ and transforming growth factor (TGF)- $\beta$; cytokines that are associated with the mobilisation of neutrophils and Th17 cells [7-13]. It is also of mechanistic interest that MATSUNAGA et al. [42] detected a trend towards a correlation between the concentration of "exhaled" IL-17A and airway obstruction in patients with asthma. Unfortunately, the utilised technique did not allow the investigators to relate exhaled IL-17A to the accumulation of luminal neutrophils [42].

\section{Bronchial tissue}

CHAKIR et al. [21] published early and important evidence for IL-17A being present in airway tissue from patients with asthma. By assessing immunoreactivity for IL-17A protein in bronchial tissue, the authors showed that patients with moderate-to-severe asthma have markedly more cells containing IL-17A protein in the sub-epithelial layer compared with healthy control subjects. Notably, there were no smokers among the patients or control subjects and none of the patients were treated with glucocorticoids [21]. The authors also found an increased IL-17A signal when analysing the intraepithelial bronchial layer [21]. The increase in IL-17A was also paralleled by a corresponding increase in TGF- $\beta$ and type I collagen, showing that the basic prerequisite for IL-17A-producing cells being involved in remodelling were present. The major part of the immunoreactivity signal for IL-17A protein was reduced by systemic treatment with a glucocorticoid, in parallel with what has previously been shown in sputum samples [36]. AL-RAmLi et al. [43] subsequently demonstrated an increase in cells immunoreactive for IL-17A protein in the sub-epithelial layer of bronchial tissue from patients with various degrees of asthma severity. In their study, AL-RAMLi et al. [43] clearly demonstrated that the magnitude of the increase in IL-17A parallels disease severity and that this increase is substantial in patients with severe asthma. For IL-17A mRNA, the pattern was similar. Unfortunately, the referred publication included very little information on the current medication of the patients.

When Doe et al. [40] recently examined submucosal cells immunoreactive for IL-17A protein they detected a trend that IL-17A is most abundant in tissue from patients with mild-to-moderate asthma when compared with tissue from healthy control subjects and patients with truly severe asthma. However, the power of the utilised material was insufficient to prove this point in a statistically valid sense and it is unclear whether there were differences in medication or smoking habits between study groups.

\section{Systemic involvement}

WONG et al. [20] were actually earlier than MOLET et al. [19] in specifically addressing the presence of IL17A protein in a study in patients with asthma. However, WONG et al. [20] examined blood cells and plasma and, thus, IL-17A at the systemic level. They found a trend towards a moderate increase in soluble IL-17A in the plasma of patients with allergic asthma when compared with healthy control subjects without allergy [20]. Unfortunately, the study material was relatively small and thus the statistical power was insufficient to prove significance. The preliminary finding by WONG et al. [20] was subsequently confirmed by the results of the study by MOLET et al. [19]; including a statistically significant increase in blood eosinophils immunoractive for IL-17A protein in patients with mild asthma compared with healthy control subjects.

Interestingly, AGACHE et al. [44] recently forwarded evidence for high IL-17A protein in serum constituting an independent risk factor for severe asthma. These investigators found that patients with severe asthma, some treated with a high dose of an inhaled glucocorticoid, have substantially more IL-17A protein in serum compared with patients with mild-to-moderate asthma. In this study, however, patients with or without atopy were not consistently separated in the analysis. The most interesting finding by AGACHE et al. [44] was that a serum IL-17A concentration exceeding a threshold $\left(20 \mathrm{pg} \cdot \mathrm{mL}^{-1}\right)$ is associated with a more than three-fold increased risk for severe disease. Here, there was evidence that serum IL-17A protein is negatively correlated with the fraction of blood neutrophils, as well as with airflow in the small airways according to spirometry. Based upon this, it can be speculated that IL-17A produced and released in the airways does recruit neutrophils from the blood, thereby contributing to small airway obstruction. Thus, the study supports the idea that IL-17A is critically involved in the pathogenesis of severe asthma.

When analysing plasma, ZHAO et al. [45] obtained evidence that IL-17A protein at the systemic level is markedly (more than five-fold) increased in patients with allergic asthma in general and even further increased in allergic patients with severe asthma in comparison to healthy control subjects. A corresponding and clear increase in the release of IL-17A protein was detected in conditioned media from peripheral blood mononuclear cells cultured and activated in vitro. From a mechanistic point-of-view, it is interesting that ZHAO et al. [45] were able to demonstrate a positive correlation between IL-17A and IL-23 protein in the group of patients with allergic asthma, since this is compatible with IL-23 constituting a trigger of IL-17A production in circulating Th17 cells. 
In a recently published study on blood Th cells from healthy control subjects and patients with moderateto-severe asthma who had been without treatment with glucocorticoids for 4 weeks, NANZER et al. [46] found that the intracellular immunoreactivity for IL-17A and IL-22 was enhanced in patients with "steroidrefractory" but not "steroid-sensitive" clinical disease. The blood Th cells co-expressing IL-17A and IL-22 were enhanced in all patients with asthma, as was the corresponding release of extracellular protein in vitro. For patients with steroid-refractory clinical disease, the release of IL-17A protein was higher than in the patients with steroid-sensitive disease. Moreover, treatment with a glucocorticoid enhanced the extracellular release of IL-17A protein in blood Th cells from healthy control subjects but not in the corresponding cells from patients with asthma, albeit the latter cells displayed more release of IL-17 in absolute terms. Clearly, this provides evidence that cytokine production in Th17 cells may be less sensitive to the inhibitory effect of glucocorticoids and, possibly, the Th17 phenotype may even be driven by this class of anti-inflammatory drugs.

\section{Multiple cellular sources \\ Sources in the lungs}

MolET et al. [19] published the initial evidence for IL-17A in eosinophils harvested from BAL and sputum samples. This evidence was obtained using three different techniques: immunocytochemistry, Western blot and in situ hybridisation [19]. Until recently, there was no functional evaluation of this potentially important finding. However, there is now a study from KOBYASH et al. [47] suggesting that isolated human blood eosinophils harvested from healthy volunteers can release IL-17A protein, albeit in response to crystals of monosodium, a stimulus of unclear relevance for human asthma.

Most evidence from human cell models, as well as from animal in vivo and in vitro models, suggests that CD3-positive mononuclear cells constitute important IL-17A- producing cells, thus pointing out T-cells [7-14, 48-50]. However, the corresponding evidence from human lungs is much more limited. In an early study, IVANOv et al. [49] demonstrated immunoreactivity for IL-17A protein in BAL cells with a lymphocyte-like morphology after exposure to organic dust in healthy human volunteers. IvANOV et al. [49] also demonstrated a substantial increase in detectable mRNA for IL-17A among BAL cells after exposure to organic dust in the same study on humans.

More recently, GLADER et al. [50] demonstrated the presence and increase of CD3- and CD4-positive but CD8-negative cells containing IL-17A in BAL samples harvested after exposure to endotoxin in a bronchial segment of healthy volunteers. These IL-17A-containing Th cells were detected in a mixed population of BAL cells; one that also contained mRNA for RORC variant 2, the archetype human Th17 transcription factor. GLADER et al. [50] also showed that the level of mRNA for RORCvariant2 correlates with the level of mRNA for IL-17A after local endotoxin exposure. The findings clearly support the idea that Th17 cells constitute an important source for IL-17A in human airways, even if this type of evidence for a Th17-like phenotype of cells has not yet been generated in patients with asthma.

PURWAR et al. [51] published evidence for Th cells producing IL-17A protein in human lungs; more precisely, CD4-positive cells containing IL-17A protein in lung tissue from patients undergoing surgery for lung cancer. The study by PURWAR et al. [51] also demonstrates that whereas the IL-17A-containing CD4positive cells constitute around $1 \%$ of all CD4-positive cells, IL-17A-containing CD8-positive cells constitute $0.8 \%$ of all CD8-positive cells in lung tissue from patients undergoing surgery for lung cancer. Thus, these findings are compatible with Tc17, as well as Th17 cells constituting sources of IL-17A in human lungs, but it is not known whether asthma is linked to both or either of these T-cell subsets.

Given the limited data on the phenotype of T-cells producing IL-17A in the airways of patients with asthma, it is important to pursue more elaborate studies in this field. The study by BuLLENS et al. [38] demonstrated a positive and relatively strong correlation between the levels of mRNA for IL-17A and CD3- $\gamma$ in sputum harvested from atopic and non-atopic patients with moderate-to-severe asthma. This correlation is compatible with the observation of AL-RAMLI et al. [43] who described immunoreactivity for IL-17A protein in mononuclear inflammatory cells, mainly residing in the bronchial submucosa of patients with asthma. It remains to be evaluated whether Th17 or Tc17 cells in the airways of patients with asthma share characteristics with the corresponding blood cells [12, 13, 48, 51-57]. Moreover, the documentation of expression for IL-17A in invariant natural killer T-cells and in mucosal-associated invariant T-cells from human subjects is intriguing but further study is needed given that there are no published studies addressing these cell types in the airways of patients with asthma [11, 13, 42, 44, 48, 50-66].

Finally, immunoreactivity for IL-17A protein has been detected in macrophages from the airways of patients with allergic asthma and this motivates more in-depth investigations [58]. This is also true for the recent detection of immunoreactivity for IL-17A in neutrophils from the airways of patients with cystic fibrosis [59]. 


\section{Sources in the blood}

There is support from several studies that Th17 and other immune cells in the blood from patients with allergic asthma can produce IL-17A protein under certain conditions.

HASHIMOTO et al. [60] demonstrated that isolated mononuclear cells harvested from the blood of patients with allergic asthma produce IL-17A protein in response to stimulation with anti-CD3 plus anti-CD28 antibodies and house dust mite extract. Examining peripheral blood mononuclear cells from patients with allergic asthma, ZHAO et al. [64] subsequently showed that there is a subset of CD3-positive and CD8-negative cells with immunoreactivity for IL-17A, arguing that it is the Th17 and not the Tc17 population that is involved in asthma. According to the referred study, the percentage of these Th17 cells is moderately increased in patients with allergic asthma, compared with healthy control subjects. Of note, the percentage of these presumed Th17 cells seems to increase with disease severity.

Cosmi et al. [61] generated T-cell clones from the CCR6- and CD161-positive fraction of CD4- and CD3positive peripheral blood cells and examined the cytokine content of these cells. After stimulation, these cells produced IL-17A and IL-4 as well as other archetype Th17- and Th2-cytokines. WANG et al. [62] have published similar findings. However, in an unorthodox manner, WANG et al. [62] claimed that the subset of CD4-positive cells containing IL-17A protein, as well as the Th2 cytokines IL-4, -5 and -13, is a CD45-, CCR6- and CRTH2-positive subset. According to WANG et al. [62], this subset contains both the archetype transcription factors for Th17 and Th2, RORCvar2 and GATA-3. This actually indicates the existence of a Th17/Th2 subset of memory Th cells, whether a committed lineage or not. According to the study by CosmI et al. [61], this subset of Th17/Th2 cells causes the secretion of IgE from autologous human B-lymphocytes (B-cells) in vitro, a mechanism indirectly linking IL-17A to true allergy. In their study, ZHAO et al. [64] demonstrated that there is an increased frequency of CD4-positive cells containing IL-17A protein in the blood of patients with allergic asthma in comparison to healthy control subjects. This was confirmed in the recent study by RAMIREZ-VELAZQUEZ et al. [63].

To date, there is very limited documentation of blood $\gamma \delta$ T-cells as sources of IL-17A in patients with asthma. However, a study by ZHAO et al. [64] reports evidence that the relative expression of IL-17A is increased among CD4-, CD8- and $\gamma \delta$ T-cells in patients with allergic asthma compared with healthy control subjects. The study did not contain any specific information on the relationship between extracellular concentrations and intracellular expression of IL-17A.

The fact that blood eosinophils may be capable of producing IL-17A protein has been discussed previously $[19,20,45]$. In addition to this evidence of granulocytes constituting a source of IL-17A, there is now evidence that a subset of blood neutrophils expressing the adhesion molecule CD177 contain IL-17A protein as well [63]. Even more interesting, this CD177-positive subset of neutrophils is markedly increased in patients with allergic asthma when compared with healthy subjects, and it is more abundant in moderate asthma than mild asthma. It is intriguing that the most pronounced signal for IL-17A in CD177-positive neutrophils among patients with allergic asthma was detected among those allergic to fungal allergens, again emphasising the link between IL-17A and pulmonary host defence [50].

\section{Receptor signalling}

It is has now been established in a range of cell and animal models that IL-17A acts by stimulating a receptor complex constituted by an IL-17 receptor A (IL-17RA) and IL-17 receptor C (IL-17RC) receptor sub-unit, signalling via the adaptor protein nuclear factor (NF) $-\kappa$ activator (Act1) downstream to more generic intracellular signalling compounds [13, 28, 56, 65-67]. These signalling compounds may include TNF receptor-associated factor-2, -3 and -6 , TGF-activated kinase- 1 and mitogen-activated protein kinases such as c-jun $\mathrm{N}$-terminal kinase, extracellular-regulated kinase and p38 [13, 28, 56, 67-69]. This signalling cascade leads to the activation of NF- $\mathrm{KB}$-activating kinase and the transcription factors NF- $\mathrm{\kappa B}$, CCAAT/ enhancing-binding protein- $\beta$ and $-\gamma[13,28,56,67-69]$. In addition, there is an Actl-independent pathway signalling via Janus kinase-1 and phosphatidylinositol 3-kinase including inactivation of glycogen synthase kinase-3 $\beta$.

Remarkably little is known about human pathology versus aberrations in signalling via IL-17 cytokines and this is particularly true for patients with asthma. PARK et al. [70] recently demonstrated that there is an association of single nucleotide polymorphisms on the IL-17RA gene and aspirine hypersensitivity in patients with asthma, thereby suggesting a pathogenic involvement of IL-17RA. Moreover, it was recently demonstrated that Actl is a client protein of ATPase and the chaperone protein heat shock protein (Hsp)90 in an animal in vivo model of psoriasis [71]. When the Act1-Hsp90 interaction is disrupted, Th17 signalling through IL-17A becomes attenuated and the corresponding signalling through IL-22 becomes hyperresponsive. Given the high mammalian conservation of IL-17A-related mechanisms, it can be 
speculated that a malfunction in the critical interaction between Act1 and Hsp90 contributes to altered IL17 signalling in human pathology, such as in asthma [71].

\section{Involvement of additional IL-17 cytokines}

There are two known members of the IL-17A cytokine family that are very similar to IL-17A from a structural and functional point of view, which have been detected in patients with asthma, namely IL-17F and IL-25 (formerly known as IL-17E) $[10,12,13]$. Of these two cytokines, it is IL-17F that can currently be linked to neutrophil mobilisation whereas IL-25 mainly seems to be linked to eosinophil mobilisation.

\section{Interleukin-17F}

The initial evidence that IL-17F is relevant for asthma in humans was published by KAWAGUCHI et al. [72]. They demonstrated that IL-17F protein stimulates the production and release of neutrophil-mobilising cytokines in human bronchial epithelial cells. They also demonstrated that local allergen challenge increases the signal for IL-17F mRNA among BAL cells in patients with allergic asthma [72].

Whereas IL-17F seems to act via the same IL-17RA/receptor C complex as IL-17A, it is conceptually interesting that IL-17F may be produced and released by a wider range of cells than is the case for the archetype Th17 cytokine IL-17A [10, 12, 13, 72]. Thus, the production of IL-17F appears to take place in structural cells as well as in immune cells, more specifically in epithelial cells, basophils, mast cells, monocytes and T-cells.

More recently, AL-RAmli et al. [43] reported an increase in subepithelial immunoreactivity for IL-17F protein in patients with verified asthma but with no information on atopy or current medication. The referred increase in IL-17F protein followed disease severity, as did the level of the corresponding mRNA. Interestingly, the study by AL-RAmLi et al. [43] indicated IL-17F protein in the epithelial layer and even within epithelial cells in the very same patients that expressed IL-17A in the submucosa. Thus, IL-17F and IL-17A may be expressed simultaneously.

In a recently published study, DoE et al. [40] reported an increased signal for IL-17F protein in the submucosa of bronchial tissue from patients with asthma compared to healthy control subjects. Even though their material failed to prove a statistically significant difference in this sense, the data from DoE et al. [40] suggested a trend that this expression of IL-17F protein increases with disease severity.

\section{Interleukin-25}

Whereas an early experimental study on a mouse model indicated that IL-25 may trigger steroid-resistant Th2 signalling via IL-4 and IL-13, there is still limited information on the relevance of IL-25 for the pathogenesis of patients with asthma [73].

When measuring protein concentrations in serum from patients with severe asthma compared with healthy control subjects, NADI et al. [74] found no clear difference in extracellular IL-25 protein. However, a trend towards somewhat higher IL-25 concentrations in patients with asthma was detected in whole blood. Unfortunately, no information on atopy was provided in this study. However, SEYs et al. [75] obtained evidence that high levels of mRNA for IL-25 in sputum cells, just like high mRNA levels for IL-17A, are increased in patients with stable asthma compared with healthy control subjects. However, both groups included atopic subjects so the role of allergy is difficult to evaluate for this study but, of clinical interest, a pattern of particularly high levels of mRNA for IL-25, IL-5 and IL-17A was associated with poorly controlled asthma. Moreover, TANG et al. [76] have recently shown that the extracellular concentration of IL-25 in plasma is increased in patients with allergic asthma compared with non-atopic healthy subjects but not when compared with atopic non-asthmatic subjects. In their study, the concentration of IL-25 in plasma displayed a modest, negative correlation with lung function assessed as forced expiratory volume in $1 \mathrm{~s}$ [76].

Tentatively, IL-25, with its proven link to the mobilisation of eosinophils rather than neutrophils, and its unique signalling via the IL-17receptor A/receptor B complex, remains of interest for further studies in patients with asthma [77, 78]. Based upon the few and relatively small studies conducted to date, it is possible, but not proven, that IL-25 is involved in the pathogenesis of certain phenotypes of asthma. If this turns out to be the case, the next goal should be to evaluate whether phenotypes with allergy are of particular interest for intervention with IL-25 signalling.

\section{How should we target IL-17 cytokines in asthma?}

The general strategy for pharmacotherapeutic development may appear an easy one. First, asthma is a disease where Th cells are believed to play a key role and there is evidence for an excess mobilisation of neutrophils in certain clinical phenotypes of asthma [2-9]. Secondly, there is evidence for an increase in the archetype Th17 cytokine IL-17A and its sibling IL-17F locally in the airways of patients with asthma 
[19, 70]. Finally, at least for IL-17A, there is also evidence for a corresponding increase in blood cells [19]. Given that IL-17A and IL-17F contribute to the mobilisation of neutrophils, these two cytokines, as well as the receptor complex they share, emerge as promising targets. However, it is not trivial to decide whether the cytokine signalling should be targeted at the protein or at the receptor level and this is for several reasons.

There is now a growing understanding that, just like neutrophils per se, IL-17A and IL-17F are critically involved in mammalian host defence against bacteria, fungi and, possibly, viruses [7-9, 11-13, 50]. Naturally, this latter fact constitutes a potential complication when it comes to targeting IL-17A and IL-17F, regardless of whether the protein or the shared receptor complex is targeted. Further indication for potential complications is brought forward by the novel experimental evidence from cell and animal models that IL$17 \mathrm{~A}$ is important for the resolution of the innate immune response and for providing negative feedback to avoid excess signalling through the IL-23-IL-17A axis (fig. 2) [23-26]. This may also constitute a therapeutic problem regardless of whether the cytokine signalling is targeted at the protein or the receptor level.

Even though we still lack corresponding data on the more exact cellular phenotype from patients with asthma, we know from the study by GLADER et al. [50] that a "Th17-like" population of immune cells is involved in the pulmonary host defence of healthy human subjects. This study clearly provides a rationale for characterising the immune cells producing IL-17A and IL-17F in the airways of patients with asthma more in detail. Moreover, the recently demonstrated involvement of "Th17-like" cells in communityacquired pneumonia, both at the local and the systemic level, underlines the need for caution when targeting cytokine signalling via IL-17A and IL-17F in patients at risk of infection [79]. It seems reasonable to assume that local inhibition of this cytokine signalling is a safer approach than systemic inhibition, but this remains to be proven.

\section{Clinical trials on the inhibition of IL-17 signalling in asthma}

To date, there is very little information on the clinical utility of targeting IL-17 cytokines in patients with asthma. BUSSE et al. [80] recently published a report of a clinical trial of an IL-17RA monoclonal antibody in patients with various types of asthma. Interestingly, a beneficial clinical effect was claimed for patients with inadequately controlled asthma who displayed high reversibility in response to a bronchodilator. Of note, this is in line with the finding of a higher level of luminal IL-17A protein among patients with more reversible airflow obstruction in the recent study by KAMINSKA et al. [39]. The clinically beneficial effect was defined as an improvement in the scoring of the Asthma Control Questionnaire, whereas the effect did not reach statistical significance in terms of lung function (defined as forced expiratory volume in $1 \mathrm{~s}$ ), for which a trend towards improvement was observed. Given that the main group of interest was only one out of nine sub-groups investigated, new, better-powered studies of the particular sub-group (i.e. phenotype) are warranted. Such studies may also clarify whether the postulated beneficial effect of the anti-IL-17RA antibody in highly reversible asthma really relates to an impact on neutrophil mobilisation or to other mechanisms. Another important question to address is whether the dosing of the anti-IL-17RA antibody was optimal for the patients that were studied.

In further support of conducting new studies, an anticipated increase in local infections was observed in the clinical trial by BuSSE et al. [80], and this side-effect is in need of further evaluation prior to developing this new pharmacotherapeutic principle.

\section{Conclusions and questions for the future}

The evidence from an increasing number of studies, albeit conducted on relatively few patients, suggests that cytokine signalling via IL-17A and IL-17F is involved in the pathogenesis of asthma. However, due to the fact that most studies have been conducted on mixed clinical phenotypes of asthma, the significance of this involvement remains uncertain. In particular, due to the lack of critical mechanistic evidence from patients it is not yet possible to make a firm conclusion with reference to the causative involvement of cytokine signalling via IL-17A and IL-17F in the mobilisation of neutrophils in asthma. This being said, it is very promising that a recent interventional [39] and a recent descriptive study [80] identified patients with moderateto-severe asthma and high reversibility as a clinical phenotype of particular interest. Even though these two important studies leave several important questions unanswered, this particular finding warrants further study.

It is also of interest to note that the growing body of evidence for not only pro- but also anti-inflammatory effects of IL-17A in experimental studies on neutrophil mobilisation, indicates a dual role of this archetype Th17 cytokine that questions the point of inhibiting it. This leaves us with the question of whether cytokine signalling via IL-17A and IL-17F controls the turnover of neutrophils in certain patients with asthma by increasing accumulation, as well as efferocytosis, apoptosis and the phagocytosis of neutrophils.

Additional critical questions to address in future studies of well-characterised patients with asthma include whether cytokine signalling via IL-17A and IL-17F contribute to the mobilisation of macrophages or the 
development of airway hyperresponsiveness in patients with particular phenotypes of asthma. This may be particularly interesting to address in patients with severe asthma [81]. The putative involvement and role of IL-25 is also in need of further study. A very interesting question for future studies is whether allergic and non-allergic asthma differ in terms of the specific involvement of IL-17A, IL-17F and IL-25 [13, 22, 26, 82, 83]. Another, more practical, question for future studies is whether the signalling pathways mediated by IL-17A and IL-17F are truly sensitive to drugs that are already in clinical practice, including glucocorticoids [36]. This question has now become even more interesting due to recent findings on the involvement of IL-17A in induced glucocorticoid insensitivity in human cells cultured in vitro and in transformed bronchial epithelial cells, as well as in primary monocytes $[84,85]$. Furthermore, given what is now known about the downstream intracellular signalling caused by IL-17A and IL-17F via the IL-17A/C receptor complex, it is imperative to establish whether this aspect of signalling is altered in patients with certain phenotypes of asthma [13, 67-69]. The same is true for IL-25 and its signalling via the IL-17RA/RB complex [77, 78].

Finally, given the now established role of IL-17A and IL-17F in pulmonary host defence, it is important to design future trials on the concept of inhibiting cytokine signalling via IL-17 cytokines so that immunosuppressive side-effects can be carefully monitored.

\section{Acknowledgements}

Careful proof reading of the manuscript by Bettina Levänen (Unit for Lung and Airway Research, Institute of Environmental Medicine, Karolinska Institutet, Stockholm, Sweden) is gratefully acknowledged.

\section{References}

1 Masoli M, Fabian D, Holt S, et al. The global burden of asthma: executive summary of the GINA Dissemination Committee report. Allergy 2004; 59: 469-478.

2 Fahy JV, Kim KW, Liu J, et al. Prominent neutrophilic inflammation in sputum from subjects with asthma exacerbation. J Allergy Clin Immunol 1995; 95: 843-852.

Wenzel SE. Asthma: defining of the persistent adult phenotypes. Lancet 2006; 368: 804-813.

4 Bhakta NR, Woodruff PG. Human asthma phenotypes: from the clinic, to cytokines, and back again. Immunol Rev 2011; 242: 220-232.

5 The ENFUMOSA cross-sectional European multicentre study of the clinical phenotype of chronic severe asthma. European Network for Understanding Mechanisms of Severe Asthma. Eur Respir J 2003; 22: 470-477.

6 Chanez P, Wenzel S, Anderson GP, et al. Severe asthma in adults: what are the important questions? J Allergy Clin Immunol 2007; 119: 1337-1348.

Lindén A, Hoshino H, Laan M. Airway neutrophils and interleukin-17. Eur Respir J 2000; 15: 973-977. Lindén A. Role of interleukin-17 and the neutrophil in asthma. Int Arch Allergy Immunol 2001; 126: 179-184. Lindén A, Adachi M. Neutrophilic airway inflammation and IL-17A. Allergy 2002; 57: 769-775.

Kolls J, Lindén A. Interleukin-17A family members and inflammation. Immunity 2004; 21: 467-476.

Lindén A, Laan M, Anderson GP. Neutrophils, interleukin-17A and lung disease. Eur Respir J 2005; 25: 159-172. Ivanov S, Lindén A. Th-17A cells in the lungs? Expert Rev Respir Med 2007; 1: 279-293.

Ivanov S, Lindén A. Interleukin-17A as a drug target in human disease. Trends Pharmacol Sci 2009; 30: 95-103.

4 Lajoie S, Lewkowich IP, Suzuki Y, et al. Complement-mediated regulation of the interleukin 17A axis is a central genetic determinant of the severity of experimental allergic asthma. Nat Immunol 2010; 11: 928-935.

15 Laan M, Cui ZH, Hoshino H, et al. Neutrophil recruitment by human IL-17A via C-X-C chemokine release in the airways. J Immunol 1999; 162: 2347-2352.

16 Hoshino H, Lötvall J, Skoogh BE, et al. Neutrophil recruitment by IL-17A into rat airways in vivo: role of tachykinins. Am J Respir Crit Care Med 1999; 159: 1423-1428.

17 Hoshino H, Laan M, Sjöstrand M, et al. Increased elastase and myeloperoxidase activity associated with neutrophil recruitment by IL-17A in airways in vivo. J Allergy Clin Immunol 2000; 105: 143-149.

18 Prause O, Bozinovski S, Anderson GP, et al. Increased matrix metalloproteinase-9 concentration and activity after stimulation with interleukin-17 in mouse airways. Thorax 2004; 59: 313-317.

19 Molet S, Hamid Q, Davoine, F, et al. IL-17A is increased in asthmatic airways and induces human bronchial fibroblasts to produce cytokines. J Allergy Clin Immunol 2001; 108: 430-438.

20 Wong CK, Ho CY, Ko FW, et al. Proinflammatory cytokines (IL-17, IL-6, IL-18 and IL-12) and Th cytokines (IFN- $\gamma$, IL-4, IL-10, and IL-13) in patients with allergic asthma. Clin Exp Immunol 2001; 125: 177-183.

21 Chakir J, Shannon J, Molet S, et al. Airway remodeling-associated mediators in moderate to severe asthma: effect of steroids on TGF- $\beta$, IL-11, IL-17A, and type I and type III collagen expression. J Allergy Clin Immunol 2003; 111 : $1293-1298$.

22 Sergejeva S, Ivanov S, Lötvall JA, et al. IL-17A as a recruitment and survival factor for airway macrophages in allergic airway inflammation. Am J Respir Cell Mol Biol 2005; 33: 248-253.

23 Silverpil E, Glader PG, Lindén A. Impact of interleukin-17 on macrophage phagocytosis of apoptotic neutrophils and particles. Inflammation 2011; 24: 1.

24 Silverpil E, Wright AK, Hansson M, et al. Negative feedback on IL-23 exerted by IL-17A during airway inflammation. Innate Immun 2013; 49: 479-492.

25 Smith E, Stark MA, Zarbock A, et al. IL-17A inhibits the expansion of IL-17A-producing T cells in mice through "short-loop" inhibition via IL-17 receptor. J Immunol 2008; 181: 1357-1364.

26 Nakae S, Komiyama Y, Nambu A, et al. Antigen-specific T cell sensitization is impaired in IL-17A-deficient mice, causing suppression of allergic cellular and humoral responses. Immunity 2002; 17: 375-387.

27 Kudo M, Melton AC, Chen C, et al. IL-17A produced by $\alpha \beta \mathrm{T}$ cells drives airway hyper-responsiveness in mice and enhances mouse and human airway smooth muscle contraction. Nat Med 2012; 18: 547-554. 
Al-Ramli W, Préfontaine D, Chouiali F, et al. Th17-associated cytokines (IL-17A and IL-17F) in severe asthma. J Allergy Clin Immunol 2009; 123: 1185-1187.

44 Agache I, Ciobanu C, Agache C, et al. Increased serum IL-17A is an independent risk factor for severe asthma. Respir Med 2010; 104: 1131-1137.

45 Zhao Y, Yang J, Gao Y, et al. Th17 immunity in patients with allergic asthma. Int Arch Allergy Immunol 2010; 151: 297-307.

46 Nanzer AM, Chambers ES, Ryanna K, et al. Enhanced production of IL-17A in patients with severe asthma is inhibited by $1 \propto, 25$-dihydroxyvitamin D3 in a glucocorticoid-dependent fashion. J Allergy Clin Immunol 2013; 132: 297-304.

47 Kobyashi T, Kouzaki H, Kita H. Human eosinophils recognize endogenous danger signal crystalline uric acid and produce proinflammatory cytokines mediated by autocrine ATP. J Immunol 2010; 184: 6350-6358.

48 Yao Z, Painter SL, Fanslow WC, et al. Human IL-17A: a novel cytokine derived from T cells. J Immunol 1995; 155: 5483-5486.

49 Ivanov S, Palmberg L, Venge P, et al. Interleukin-17A mRNA and protein expression within cells from the human bronchoalveolar space after exposure to organic dust. Respir Res 2005; 6: 44.

50 Glader P, Smith ME, Malmhäll C, et al. Interleukin-17-producing T-helper cells and related cytokines inhuman airways exposed to endotoxin. Eur Respir J 2010; 36: 1155-1164.

51 Puwar R, Campbell J, Murphy G, et al. Resident memory T cells (TRM) are abundant in human lung: diversity, function and antigen specificity. PLoS One 2011; 6: e16245.

52 Aarvak T, Chabaud M, Miossec P, et al. IL-17A is produced by some proinflammatory Th1/Th0 cells but not by TH2 cells. J Immunol 1999; 162: 1246-1251.

53 Hoeve MA, Savage NDL, de Boer T, et al. Divergent effects of IL-12 and IL-23 on the production of IL-17A by human T cells. Eur J Immunol 2006; 36: 661-670.

54 Acosta-Rodriguez EV, Rivino L, Geginat J, et al. Surface phenotype and antigenic specificity of human interleukin17A-producing T helper memory cells. Nat Immunol 2007; 8: 639-646.

55 Acosta-Rodriguez EV, Napolitani G, Lanzavecchia A, et al. Interleukins $1 \beta$ and 6 but not transforming growth factor- $\beta$ are essential for the differentiation of interleukin 17-producing human T helper cells. Nat Immunol 2007; 8: 942-949.

56 Wilson NJ, Boniface K, Chan JR, et al. Development, cytokine profile and function of human interleukin 17-producing helper T cells. Nat Immunol 2007; 8: 950-957.

57 Annunziato F, Cosmi L, Santarlasci V, et al. Phenotypic and functional features of human Th17 cells. J Exp Med 2007; 204: 1849-1861.

58 Song C, Luo L, Lei Z, et al. IL-17A-producing alveolar macrophages mediate allergic lung inflammation related to asthma. J Immunol 2008; 181: 6117-6124.

59 Brodlie M, McKean MC, Johnson GE, et al. Raised interleukin-17 is immunolocalised to neutrophils in cystic fibrosis lung disease. Eur Respir J 2011; 37: 1378-1385.

60 Hashimoto T, Akiyama K, Kobyashi N, et al. Comparison of IL-17A production by helper T cells among atopic and nonatopic asthmatics and control subjects. Int Arch Allergy Clin Immunol 2005; 137: 51-54.

61 Cosmi L, Maggi L, Santarlasci V, et al. Identification of a novel subset of human circulating memory CD4 ${ }^{+} \mathrm{T}_{\text {cells }}$ that produce both IL-17A and IL-4. J Allergy Clin Immunol 2010; 125: 222-230.

62 Wang YH, Voo KS, Liu B, et al. A novel subset of CD4+ Th2 memory/effector cells that produce inflammatory IL-17A cytokine and promote the exacerbations of chronic allergic asthma. J Exp Med 2010; 207: 2479-2491. 
Ramirez-Velazquez C, Castillo EC, Guido-Bayardo L, et al. IL-17-producing peripheral blood CD $177^{+}$neutrophils increase in allergic asthmatic subjects. Allergy Asthma Clin Immunol 2013; 9: 23.

64 Zhao Y, Yang J, Gao YD. Altered expressions of helper T cell (Th1), Th2, and Th17 cytokines in CD8 ${ }^{+}$and $\gamma \delta$ T cells in patients with allergic asthma. J Asthma 2011; 48: 429-436.

65 Le Bourhis L, Mburu YK, Lantz O. MAIT cells, surveyors of a new class of antigen: development and functions. Curr Opin Immunol 2013; 25: 174-180.

66 Rachitskaya AV, Hansen AM, Horai R, et al. Cutting edge: NKT cells constitutively express IL-23 receptor and ROR $\gamma t$ and rapidly produce IL-17 upon receptor ligation in an IL-6-dependent fashion. J Immunol 2008; 180: $167-171$.

67 Shen F, Gaffen SL. Structure-function relationships in the IL-17A receptor: implications for signal transduction and therapy. Cytokine 2008; 41: 92-104.

68 Gaffen SL. Structure and signalling in the IL-17 receptor family. Nat Rev Immunol 2009; 9: $556-567$.

69 Lindén A. A role for the cytoplasmic adaptor protein Actl in mediating IL-17 signalling. Sci STKE 2007; 398: re4.

70 Park JS, Park BL, Kin MO, et al. Association of single nucleotide polymorphisms on interleukin 17 receptor A (IL17RA) gene with aspirin hypersensitivity in asthmatics. Hum Immunol 2013; 74: 598-606.

71 Wang C, Wu L, Bulek K, et al. Psoriasis-associated variant Act1 D10N with impaired regulation by Hsp90. Nat Immunol 2013; 14: 72-81.

72 Kawaguchi M, Onuchic LF, Li XD, et al. Identification of a novel cytokine, ML-1, and its expression in subjects with asthma. J Immunol 2001; 167: 4430-4435.

73 Petersen BC, Budelsky AL, Baptist AP, et al. Interleukin-25 induces type 2 cytokine production in a steroid resistant interleukin-17RB+ myeloid population that exacerbates asthmatic pathology. Nat Med 2012; 18: 751-758.

74 Nadi E, Arjipor M, Sharifi S, et al. Assay of IL-22 and IL-25 in serum, whole blood, and peripheral blood mononuclear cell cultures of patients with severe asthma. Allergol Immunopathol (Madr) 2014; 42: 402-406

75 Seys SF, Grabowski M, Adriaensen W, et al. Sputum cytokine mapping reveals an "IL-5, IL-17A, IL-25-high" pattern associated with poorly controlled asthma. Clin Exp Allergy 2013; 43: 1009-1017.

76 Tang W, Smith SG, Beaudin S, et al. IL-25 and IL-25 receptor expression on eosinophils from subjects with allergic asthma. Allergy Immunol 2014; 163: 5-10.

77 Lee J, Ho WH, Marouka M, et al. IL-17E: a novel proinflammatory ligand for the IL-17 receptor homolog IL-17Rh1. J Biol Chem 2001; 276: 1660-1664.

78 Rickel EA, Siegel LS, Yoon BR, et al. Identification of functional roles for both IL-17RB and IL-17RA in mediating IL-25-induced activities. J Immunol 2008; 181: 4299-4319.

79 Paats MS, Bergen IM, Hanselaar WE, et al. T helper 17 cells are involved in the local and systemic inflammatory response in community-acquired pneumonia. Thorax 2013; 68: 468-474.

80 Busse WW, Holgate ST, Kerwin EM, et al. Randomized double-blind, placebo-controlled study of brodalumab, a human anti-IL-17 receptor monoclonal antibody, in moderate to severe asthma. Am J Respir Crit Care Med 2013; 188: 1294-1302.

81 Wenzel SE. Asthma phenotypes: the evolution from clinical to molecular approaches. Nat Med 2012; 18: 716-725.

82 Hellings PW, Kasran A, Liu Z, et al. Interleukin-17A orchestrates the granulocyte influx into airways after allergen inhalation in a mouse model of allergic asthma. Am J Respir Cell Mol Biol 2003; 28: 42-50.

83 Oda N, Canelos PB, Essyan DM, et al. Interleukin-17F induces pulmonary neutrophilia and amplifies antigen induced allergic response. Am J Respir Crit Care Med 2005; 171: 12-18.

84 Zijlstra GJ, ten Hacken NH, Hoffmann RF, et al. Interleukin-17A induces glucocortiocoid insensitivity in human bronchial epithelial cells. Eur Respir J 2012; 39: 439-445.

85 Vasquez-Tello A, Halwani R, Hamid Q, et al. Glucocorticoid receptor- $\beta$ up-regulation and steroid resistance induction by IL-17 and IL-23 cytokine stimulation in peripheral mononuclear cells. J Clin Immunol 2013; 33: $466-478$. 